\title{
Ein Lesebuch zur Psychiatrie
}

\section{Michael Soyka}

Prof. Dr. med., Facharzt für Psychiatrie und Psychotherapie, Ärztlicher Direktor Privatklinik Meiringen

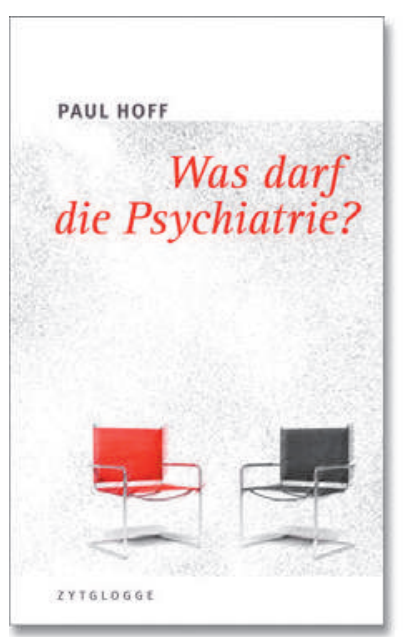

Paul Hoff

Was darf

die Psychiatrie?

Oberhofen:

Zytglogge-Verlag; 2014.

240 Seiten. 36 CHF.

ISBN 978-3-7296-0886-3

Paul Hoff, stellvertretender Direktor der PUK Zürich und ein profilierter Kenner der deutschsprachigen Psychiatrie mit herausragender Expertise - vor allem in der Psychopathologie und der forensischen Psychiatrie - legt ein interessantes, schon im Titel kontroverses Buch vor: Was darf die Psychiatrie? Oder anders ausgedrückt: Was darf der Psychiater? Und: Was können und dürfen Diagnostik, Therapie, Forschung und, nicht überraschend, beim wissenschaftlichen Schwerpunkt von Paul Hoff, die Begutachtung, sprich die forensische Psychiatrie?

In seinem Buch geht Paul Hoff zunächst der Frage nach, wo die Psychiatrie heute steht. Er beschäftigt sich mit ihren ethischen Grundlagen, schlägt einen weiten Bogen von Hippokrates über Kant bis zu modernen Autoren und flicht interessante Fallvorstellungen wie z.B. die «von einem schizophrenen Lebenskünstler» in seine Überlegungen und Argumentationskette ein.

Im Diagnostik-Kapitel geht er der für die Psychiatrie besonders relevanten Frage nach, was krank ist und was nicht. Hier schlägt er den Bogen von Carl Jaspers zu den neuen Klassifikationssystemen wie ICD-10 und DSM-5 mit ihren zuletzt «über»-detailreichen Definitionen psychischer Störungen.

Ausführlich diskutiert wird auch die angebliche Theorielosigkeit der modernen psychiatrischen Klassifikationssysteme. Kurz werden aktuelle Probleme wie z.B. die Rolle von Biomarkern als Indikatoren psychischer Erkrankungen (praktisches Beispiel: EEG-Befunde bei psychiatrischen Begutachtungen für die Invalidenver- sicherung!) angesprochen. Nachvollziehbar spricht Hoff von Biomarkern als kleinen, aber wahrscheinlich relevanten Teilen eines grossen Puzzles, bevor er sich seiner vielleicht wichtigsten Frage zuwendet, nämlich der Rolle der Psychopathologie heute: Relikt oder Kernelement? Es folgen Ausführungen zu den verschiedenen Formen von psychiatrischen, psychotherapeutischen und sozialtherapeutischen Interventionen und der "Achillesferse» der Psychiatrie, nämlich den medizinischen Zwangsmassnahmen und der Frage der Patienten-Autonomie. Hier werden Fragen wie die Urteilsfähigkeit von Patienten oder Grundlagen fürsorgerischer Unterbringungen sowie Zwangsbehandlungen diskutiert.

In den einzelnen Kapiteln runden Fallvorstellungen die angesprochenen Problemstellungen ab.

Anschliessend formuliert Hoff zehn Thesen zur Psychiatrie, die er ausdrücklich als Teildisziplin der Medizin sieht. Allerdings verwahrt er sich auch gegen eine

Hoff verwahrt sich gegen eine «reflexartige Festlegung der Psychiatrie auf ein strikt medizinisches Krankheitsmodell».

«reflexartige Festlegung der Psychiatrie auf ein strikt medizinisches Krankheitsmodell».

Ein Fazit: Es handelt sich nicht um ein Lehrbuch der Psychiatrie, auch nicht um eine Streitschrift, sondern um ein interessantes - im wahrsten Sinne des Wortes Lesebuch zur heutigen Situation der Psychiatrie in Klinik und Forschung.

Gerne hätte man noch mehr aus Sicht des Autors erfahren, etwa wie er die Vermittlung der Psychiatrie an den Universitäten heute sieht. Hat der offenkundige Nachwuchsmangel nicht auch hier gewisse Gründe? Wer sich für die ethischen Grundlagen der Psychiatrie, ihren kultur- und geistesgeschichtlichen Hintergrund, insbesondere auch konkrete Fragen wie der Diagnostik psychiatrischer Erkrankungen und der Begutachtung bzw. Behandlung psychisch kranker Rechtsbrecher interessiert, wird in diesem schönen Buch viel Lesensund Anregenswertes finden. Nicht selbstverständlich für ein Psychiatrie-Buch heute: Es ist flüssig geschrieben, gut zu lesen und in einem exquisiten Deutsch verfasst, so wie es früher einmal in der Psychiatrie und Wissenschaft gängig war. 